\title{
TTR
}

Traduction, terminologie, rédaction

\section{James W. Underhill. Voice and Versification in Translating Poems. Ottawa, University of Ottawa Press, coll. Perspectives on Translation, 2016, 333 p.}

\section{Benoit Laflamme}

Volume 29, numéro 2, 2e semestre 2016

URI : https://id.erudit.org/iderudit/1051021ar

DOI : https://doi.org/10.7202/1051021ar

Aller au sommaire du numéro

Éditeur(s)

Association canadienne de traductologie

ISSN

0835-8443 (imprimé)

1708-2188 (numérique)

Découvrir la revue

Citer ce compte rendu

Laflamme, B. (2016). Compte rendu de [James W. Underhill. Voice and Versification in Translating Poems. Ottawa, University of Ottawa Press, coll. Perspectives on Translation, 2016, 333 p.] TTR, 29(2), 203-206.

https://doi.org/10.7202/1051021ar d'utilisation que vous pouvez consulter en ligne.

https://apropos.erudit.org/fr/usagers/politique-dutilisation/ 
continent. The book is of interest not only because it provides insights into one of the most interesting works of literature of the $19^{\text {th }}$ century, but also because students and academics alike will find in it the results of a systematic and thorough research project covering the life of a masterpiece over one and a half centuries, across four languages and several countries.

\section{References}

Brisset, Annie (2002). "Clémence Royer ou Darwin en colère." In J. Delisle, ed. Portraits de traductrices. Ottawa, University of Ottawa Press, pp. 173-203.

Pym, Anthony (1998). Method in Translation History. Manchester, St. Jerome.

\section{Marco A. Fiola RYERSON UNIVERSITY}

\section{James W. Underhill. Voice and Versification in Translating Poems. Ottawa, University of Ottawa Press, coll. Perspectives on Translation, 2016, 333 p.}

Louvrage Voice and Versification in Translating Poems se veut une réponse à l'idée convenue que la traduction de la poésie est une tâche impossible. Faisant valoir que cette intraduisibilité supposée découle d'une division erronée entre le fond et la forme en poésie (à savoir que cette division n'a pas lieu d'être), James W. Underhill aborde la question en trois temps, d'abord en déconstruisant la conception classique de la forme en poésie dans la partie "Versification", puis en la reconstruisant en tandem avec le fond dans la partie "Form and Meaning in Poetry Translation» et enfin en utilisant l'approche de traduction qu'il préconise pour commenter des traductions de Baudelaire et de Dickinson dans la dernière partie, «Case Studies».

\section{Versification}

Dans la première partie, la plus volumineuse et la plus théorique du livre, Underhill fait valoir que la forme ne doit plus être considérée comme un aspect secondaire de la poésie ou, inversement, comme la seule caractéristique de ce genre littéraire. S'appuyant sur les travaux d'Henri Meschonnic, il montre comment la voix poétique jaillit de l'alliance entre le sens et la forme. C'est-à-dire que la forme n'est ni une béquille sur laquelle s'appuie le sens ni un cadre 
préétabli dans lequel le poète doit insérer ses mots. Fond et forme sont en symbiose, l'un alimentant l'autre pour créer un tout. À ce titre, Underhill insiste bien que la forme ne peut se réduire à la simple métrique : elle comprend également d'autres structures et procédés, comme l'allitération, le rythme et l'accentuation.

Underhill procède ensuite à une analyse comparative de l'utilisation de la forme en poésie en anglais et en français, soulignant les procédés propres à chaque langue. L'analyse de l'accentuation et de son usage dans les deux langues est fort pertinente et met en lumière des différences fondamentales dans les modes d'expression poétique du français et de l'anglais (l'anglais ayant recours à la "promotion» et la "demotion» tonique des syllabes ${ }^{1}$, et le français, à la diérèse et à la synérèse).

Les deux derniers chapitres de la première partie présentent une analyse approfondie de la métrique, de la rime et d'autres structures internes des poèmes (les figures de répétition, la manipulation des syllabes, la rime interne) pour dégager leur utilité et leur effet sur le sens et la voix poétique.

\section{Form and Meaning}

Ayant bien expliqué sa conception de la forme, Underhill consacre la deuxième partie de l'ouvrage à analyser l'interaction entre fond et forme. C'est ici qu'il propose une approche à la traduction qui vise une fidélité à la fois au sens et à la forme. Pour lui, la forme ne doit pas être un carcan qui brime la voix poétique et le sens ne doit pas être la seule priorité du traducteur au point où il abandonne toute considération formelle. Tout au long de cette partie, Underhill appuie ses arguments sur des exemples de la poésie classique française et anglaise et propose une analyse des traductions pour souligner à la fois les bons réflexes de traduction et les passages qui auraient mérité plus de travail. Il y a lieu de souligner que l'auteur offre en exemple des traductions de son cru afin d'illustrer son approche où forme et fond ne sont pas deux éléments complémentaires, mais bien une seule et même entité. Comme le livre repose en grande partie sur la critique du travail de

1. Dans Poetic Rhythm, An Introduction, Derek Attridge (1995) indique que les syllabes accentuées correspondent habituellement à un temps (beat) et les syllabes inaccentuées, à un contretemps (offbeat). Toutefois, "since a stressed syllable is normally expected to function as a beat, it is said to be demoted when it occurs in an offbeat. Conversely, an unstressed syllable is said to be promoted when it functions as a beat» (p. 70; les italiques sont d'Attridge). 
nombreux traducteurs, il faut féliciter l'auteur d'oser présenter ses propres traductions. Louvrage, qui aurait pu sembler prescriptif et négatif sans ces créations personnelles, prend une tournure beaucoup plus constructive. L'approche proposée n'apparait plus servir uniquement à critiquer des traductions qu'il analyse, mais bien à en construire de plus cohérentes.

\section{Case Studies}

La dernière partie du livre est consacrée à l'analyse approfondie des différentes traductions de deux monuments de la poésie française et anglaise, Charles Baudelaire et Emily Dickinson. Avant de se lancer dans la critique et le commentaire des traductions des poèmes de Baudelaire, Underhill consacre une bonne partie du chapitre à la vie, aux thèmes et au style du poète. Bien que Baudelaire soit une figure connue de la poésie, ces précisions permettent de mieux comprendre la critique des traductions formulées en fin de chapitre.

Underhill regroupe les différents traducteurs de Baudelaire en anglais selon l'approche de traduction adoptée (stratégie archaïsante, stratégie de modernisation ancrée dans une métrique, traduction en prose, traduction en vers libres et transcréation).

Peut-être parce que l'ouvrage s'adresse avant tout à des lecteurs anglophones, la vie, les thèmes et le style d'Emily Dickinson ne sont pas explorés avec autant d'exhaustivité que dans la partie consacrée à Baudelaire. Underhill y étudie les traductions d'Emily Dickinson en français qu'ont produites Claire Malroux et Françoise Delphy et celles en allemand de Gertrud Liepe. Si les critiques que formule Underhill tout au long de l'œuvre au sujet de diverses traductions nous semblent fondées et bien étayées, les critères qu'utilise Underhill pour évaluer les traductions de Delphy dans cette partie semblent manquer de rigueur. Alors qu'il applaudit les ajouts qui caractérisent les traductions françaises produites par certains, lorsque Delphy emprunte cette même stratégie il s'agit d'une erreur. Le travail d'Underhill suppose bien sûr une dose importante de subjectivité, mais il parvient partout ailleurs dans l'ouvrage à étayer ses observations. Dans le cas de Delphy, ce travail d'explication semble lacunaire.

\section{Conclusion}

L'approche proposée par Underhill est d'un grand intérêt et s'appuie sur des fondements théoriques solides, notamment les 
écrits de Derek Attridge (p. ex. The Rhythms of English Poetry. London, Longman, 1982), de Richard Cureton (p. ex. Rhythmic Phrasing in English Verse. New York, Longman, 1992) et de Henri Meschonnic (p. ex. Poétique du traduire. Paris, Verdier, 1999). Les chapitres comportent habituellement, en conclusion, une liste de questions et d'éléments à évaluer au moment de traduire un poème. Ces listes résument brièvement les principaux éléments constitutifs des poèmes et permettent au traducteur d'orienter son analyse du poème. Nous notons d'ailleurs un glossaire de vingt pages fort pertinent en fin d'ouvrage. Cet ajout est d'une grande utilité pour le lecteur ne possédant pas de connaissances approfondies en étude de la poésie. Selon Underhill, les connaissances théoriques de la poésie font parfois défaut aux traducteurs : ce glossaire explique clairement et succinctement les grands concepts théoriques qui ont une incidence sur la traduction de la poésie.

Il faut cependant mentionner le nombre important de coquilles dans les passages et poèmes français cités (fourgueux*/ fougueux, incessant*/incessante, raconteur*/raconter, divins*/ divans). Il est en outre dommage qu'un exemple de manipulation syntaxique en français repose sur une formulation qu'un locuteur francophone n'utiliserait pas naturellement : «Je ne ferais jamais plus confiance à toi!» Enfin, il convient de souligner qu'il serait avantageux, dans une édition ultérieure, de supprimer ou d'étayer les affirmations catégoriques non expliquées (p. ex. "The French has yet to produce a playwright of [Shakespeare's] genius.») (p. 231).

Tout compte fait, l'ouvrage de James W. Underhill est un apport pertinent à l'étude de la traduction de la poésie. L'auteur y déconstruit des a priori et des mauvaises conceptions de la poésie qui empêchent les traductions de poèmes de rayonner avec autant d'éclat que dans leur version originale.

\section{Benoit Laflamme Université du Québec en Outaouais}

